International Journal of Electrical and Power Engineering 6 (1): 38-42, 2012

ISSN: 1990-7958

(C) Medwell Journals, 2012

\title{
The Increasing Energy Efficiency of the Vibratory Conveying Drives with Electromagnetic Excitation
}

\author{
Zeljko Despotovic and Aleksandar Ribic \\ Mihajlo Pupin Institute, Belgrade, Serbia
}

\begin{abstract}
The electromagnetic vibratory conveying drives provide easy control gravity flow of bulk and particulate materials. The achievement of free vibration of variable intensity and frequency in a wide range through adequate power converter and corresponding controller ensures the continuous flow of material under different conditions. Today, as a standard semiconductor output stage of power using thyristors and triacs. This implies the use of phase control. Considering that in this case, fixed frequency of power grid, changing the phase angle can be achieved by adjusting the amplitude of oscillations of the conveyor but not its frequency. Transistor converters in addition to amplitude control provided the frequency control. In this way, the excitation of transporter is independent of the main frequency. Frequency control provides operation of vibratory conveyors in the region of mechanical resonance. Working in the resonance band is energetically favorable, since then has minimal power consumption of the entire vibratory conveying drive. This study presents a concrete implemented, current controlled transistor converter used to excite resonant electromagnetic vibration conveyor and experimental results as an indicator of energy efficiency.
\end{abstract}

Key words: Vibratory conveyor, electromagnetic drive, resonance, power converter, current control, energy efficiency

\section{INTRODUCTION}

Vibratory conveyors are widely used devices for transport of granular and particulate material in various technological processes involving gravimetric transport, processing and dosing industries. Vibratory conveyors having electromagnetic drive are a very popular because of their high efficiency and easy maintenance. This drive is based on the Electromagnetic Vibratory Actuator (EVA). From the macroscopic view, the process of vibratory transport is based on micro-throws of particles of the material being conveyed (Goncharevich et al., 1990; Dyr and Wodzinski, 2002; Sloot and Kruyt, 1996; Soto-Yarritu and Martinez, 2001). Vibrations of the vibratory trough, i.e., Load Carrying Element (LCE), containing the material, cause motion of material particles. Therefore, the material obtains the character of a viscous fluid and as becomes suitable for conveying or further processing. By realizing free vibrations of variable amplitude and frequency over a wide range through application of the EVA, suitable power converter and the corresponding controller, continuous conveyance and dosing of granular materials have been provided for various operating conditions. In this way, the whole vibratory conveying system has a behavior of the controllable mechanical oscillator (Doi et al., 1999, 2001).
Standard power output stages intended for control of vibratory conveyance using thyristors and triacs imply the use of phase angle control. Since, the supply network frequency is constant $(50 / 60 \mathrm{~Hz})$, phase angle variation can only accomplished tuning of amplitude oscillations of the LCE. Its oscillation frequency cannot be adjusted by these converters. Application of transistor switching converters enables accomplishing the amplitude and/or frequency control of vibratory conveying. Their use makes the excitation of a vibratory conveyor independent of the supply network frequency. In addition, the frequency control ensures operation of vibratory conveyors in the region of mechanical resonance. Operation in the resonance region is becoming very energy efficient because large output displacement of LCE is provided by small input power. In other words in this case, the entire vibratory system has low power consumption.

\section{THE RESONANT VIBRATORY CONVEYOR WITH ELECTROMAGNETIC DRIVE}

For most technical systems work in the resonance range is unfavorable because it can lead to significant stresses and fracture mechanical construction since in this case for small values of excitation forces get very

Corresponding Author: Zeljko Despotovic, Mihajlo Pupin Institute, Belgrade, Serbia 


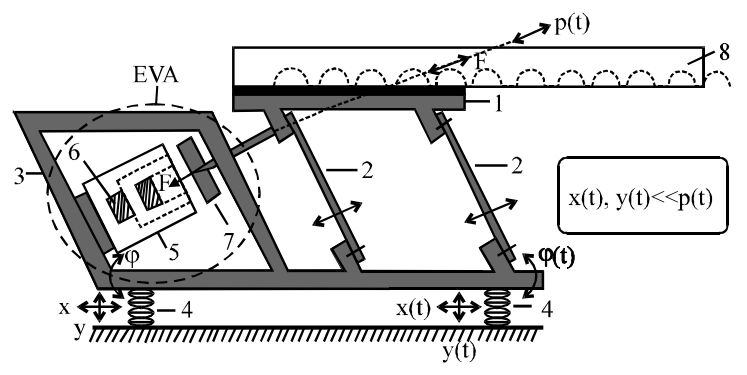

Fig. 1: Typically construction of Electromagnetic Resonant Vibratory Conveyor (ERVC)

large oscillation amplitudes. Limit the amplitude can be achieved by appropriate control of excitation force. In this case, work in the resonant range becomes advantageous since large enough but the final value of the amplitude of the oscillation can be obtained forrelatively small energy of excitation. This is especially important from the point of using Electromagnetic Resonant Vibratory Conveyor (ERVC). A typical arrangement of ERVC is shown in Fig. 1. Its main components are: the load carry element-1, EVA as source of excitation force $\mathrm{F}$ and flexible element-2. Flexible elements are made of composite leaf (fiberglass) springs. These elements are rigidly connected with the LCE on their one side while on the other side, they are fitted to the base- 3 of the device and sloped down under certain angle. The base of ERVC is resting on rubber pads-4 to the foundation. EVA consists of a magnetic core- 5 covered by continuous windings coil- 6 . As the ferromagnetic material has a very high permeability, all energy of the magnetic field is stored in air gap. Created magnetic field producing the electromagnetic force $\mathrm{F}$ acts on armature-7 attached to the LCE. This element carries the vibratory trough- 8 along with conveying material. Displacement $\mathrm{p}(\mathrm{t})$ of vibratory trough is dominant and much larger then vertical and horizontal displacements of base $\mathrm{x}(\mathrm{t})$ and $\mathrm{y}(\mathrm{t})$, respectively.

Taking into account, the dominant influence flexible elements (whose characteristics are: stifness-k and damping- $\beta$ ) on the oscillations of vibrating trough whose mass $\mathrm{m}$, it is possible to provide a mechanical system with four Degrees of Freedom (4 DOF) with state variable p, $x$, $y, \varphi$ represent with a simplified mechanical system with 1 DOF as shown in Fig. 2. Detailed dynamic model of ERVC is given by Doi et al. $(1999,2001)$, taking into account several oscillating modes but all of them are not interesting for further analysis. The typical amplitude-frequency characteristics of ERVC are shown on Fig. 3. The characteristic-1 refers to a complete model of ERVC while characteristic-2 refers to its simplified representation.

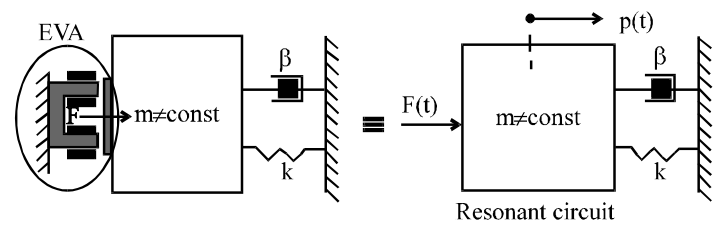

Fig. 2: Simplified presentation of ERVC

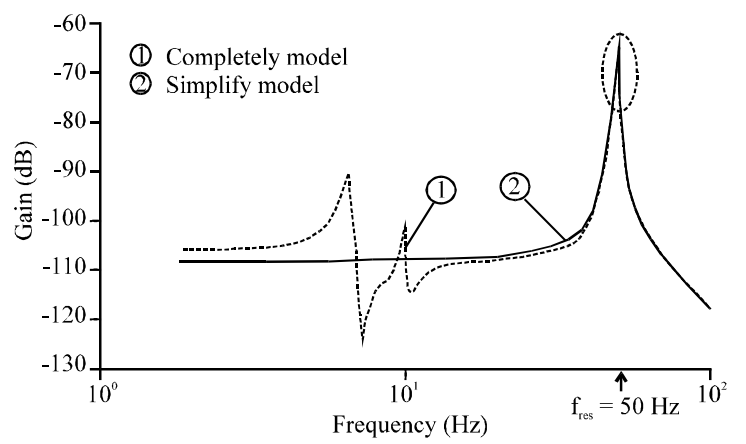

Fig. 3: Typical amplitude-frequency characteristic of ERVC

In the frequency range of $20-100 \mathrm{~Hz}$, these two characteristics are very well coincide which justifies the previous approximation. The optimal vibratory transport is being obtained for frequency within the range $20-150 \mathrm{~Hz}$ and vibratory width (i.e., double amplitude oscillation of LCE) range $0.1-20 \mathrm{~mm}$ for the most of materials (Goncharevich et al., 1990; Parameswaran and Ganapahy, 1979). The pulse excitation is from the energy point of view, the most suitable method of excitation of ERVC. The generating pulses of excitation force $\mathrm{F}$ that are in resonance with the mechanical part of the system can be achieved trough current pulses of EVA. This mechanical excitation force is proportional to the square current of EVA, i.e., F i² (Goncharevich et al., 1990; Doi et al., 1999; Despotovic and Stojiljkovic, 2007).

\section{THE SWITCHING CONVERTERS FOR CONTROL ERVC}

From electrical standpoint, EVA is mostly inductive load by its nature, so that simply generating the sinusoidal or triangle half-wave current is possible by power converter. Application of ERVC in combination with power converters provides flexibility during work. The using phase controlled thyristor or triac converters in vibratory conveyance, implies a fixed frequency of vibrations, imposed by the supply network frequency (Doi et al., 2001; Dewan and Straughen, 1975; Despotovic and Stojiljkovic, 2005, 2007). A serious problem arises when the mass of the conveying material is changed, i.e., mechanical resonance of the system has changed. In such 
case, the vibratory system will not operate efficiently. It is possible to tune amplitude but not the frequency of the vibrations. In addition, the thyristor converter brings in a DC component and undesirable higher harmonics. Application of triacs results in somewhat better as regards the harmonic content but the same problem arises if the resonant frequency is changed. Variation of the mechanical resonance frequency due to variation of the mass of the conveyed material or even variation of the system parameters (drift of the characteristic springs, stiffness, etc.) leads to reduction of efficiency of the vibratory drives. In order to accomplish an optimal and efficient operation at a new resonant frequency, it is necessary to change the frequency of EVA current. The work on application switching transistor converters for obtaining current pulses through EVA coil has been intensified in recent times. Mainly, the three topologies have been accepted in practice (Sokolov et al., 2007; Despotovic and Ribic, 2009). These topologies can be achieved by different excitation current waveforms (sinusoidal, triangle, etc.). The required sine-wave (half-wave) EVA current can be realized by these topologies if the applied current controls which is basedon tracking the reference value with adjustable time duration amplitude and frequency. This method of generation of the excitation current has the advantage in that it allows independent tuning of the frequency and amplitude of the electromagnetic excitation force $\mathrm{F}$.

The switching converter described by Despotovic and Stojiljkovic $(2005,2007)$ despite its advantages, suffers from a serious shortcoming that at high frequencies its switching losses become dominant. Also, the losses in iron of the magnetic circuit and in copper of EVA coil become also significant. This reduces the efficiency of the ERVC and it is not unusual that the power losses in the converter are higher than the power required for maintaining the resonant oscillatory mode. By a suitable control, it is possible to overcome this problem and accomplish the expected vibratory effect, i.e., the required amplitude of LCE oscillations and optimal operating frequency of the ERVC. The half bridge topology which provides simply control of duration, amplitude and frequency of the triangular current halfwave is shown in Fig. 4. Detailed analysis and operation of this topology are presented by Mohan et al. (1995), Rashid (1988) and Ribic and Despotovic (2010).

The principal diagram of the amplitude-frequency control of the ERVC and measuring of characteristic values are shown in Fig. 5. EVA coil voltage is determined by the control signal $u_{D}$. As a result of tuning the width of the control voltage $u_{D}$, i.e., the output voltage of the bridge converter, a triangular waveform of the current

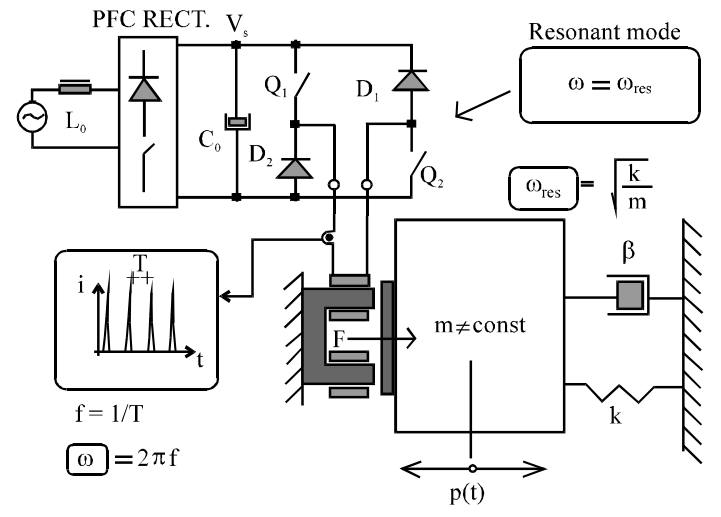

Fig. 4: Half bridge topology for control of ERVC

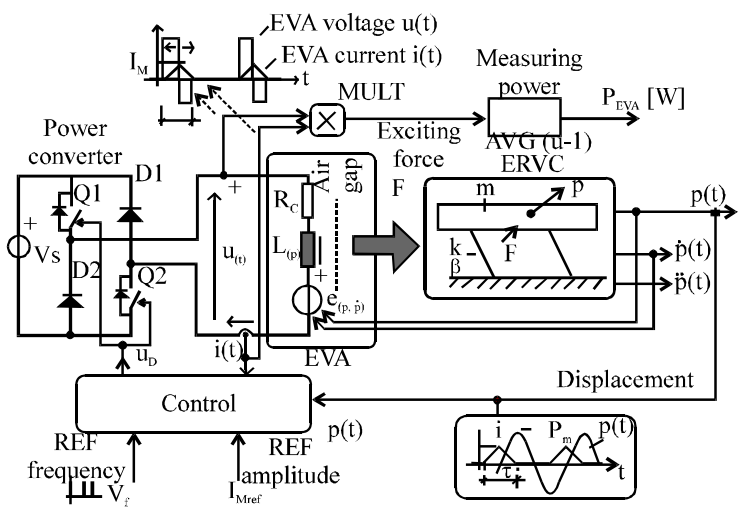

Fig. 5: Block diagram of control and measuring of ERVC

which supplying EVA coil is generated. The current pulse is created in the way that the current pulse peak corresponds at the instant when the armature passes through the equilibrium position, i.e., when $\mathrm{p}=\mathrm{p}_{0}$, therefore the inductance of EVA coil in this case is $\mathrm{L}(\mathrm{p})=\mathrm{L}_{0}$ (Ribic and Despotovic, 2010).

The amplitude control, i.e., adjustment of the output displacement $\mathrm{p}(\mathrm{t})$ is achieved by changing the amplitude and time duration of triangle half-wave current pulses of the EVA.

\section{RESULTS AND DISCUSSION}

The experimental results are obtained on really realized prototype ERVC in which applied the programmed current control as shown in Fig. 5. The measured values are interest: current EVA-i(t), output displacement of ERVC-p(t) and power consumption of EVA- $P_{\mathrm{EVA} A}$. The amplitude control is confirmed by oscilloscopic records as shown in Fig. 6 for two values of amplitude and duration current pulses. The records are obtained for DC link voltage $\mathrm{V}_{\mathrm{s}}=380 \mathrm{~V}$. The 
Int. J.Elec. Power Eng., 6 (1): 38-42, 2012
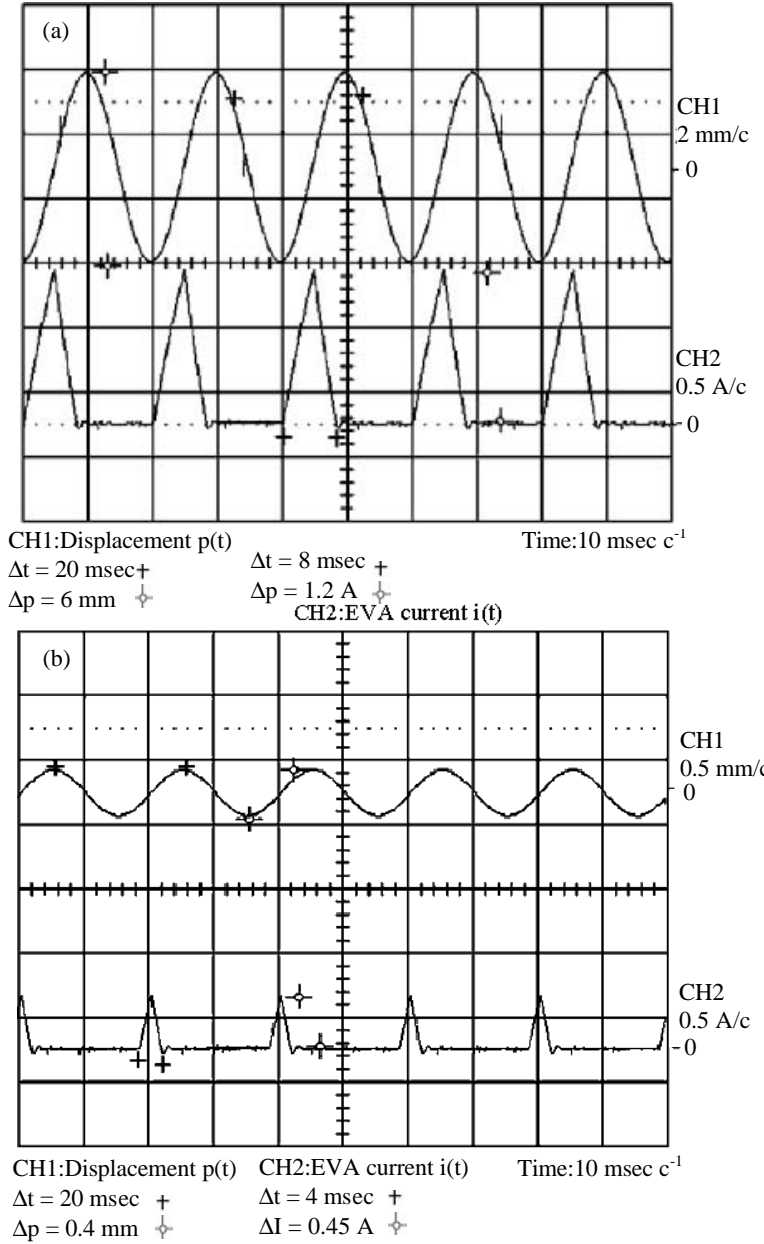

Fig. 6: Amplitude control of output displacement of ERVC by programmed current control of EVA; a) vibratory width $P_{p-p}=6 \mathrm{~mm}$; b) vibratory width $\mathrm{P}_{\mathrm{p}-\mathrm{p}}=4.0 \mathrm{~mm}$

frequency of the outputcurrent is set to $\mathrm{f}=50 \mathrm{~Hz}$ which is closely of mechanical resonant frequency. The mechanical parameters of ERVC have had following values: total mass of load carrying element $m=1.15 \mathrm{~kg}$ and the stiffness of fiberglass springs $\mathrm{k}=114 \mathrm{~N} \mathrm{~mm}^{-1}$ accordingly, the mechanical resonance frequency was $\mathrm{f}_{\text {res }}=50.3 \mathrm{~Hz}$. The oscilloscopic record of EVA current and output displacement $\mathrm{p}(\mathrm{t})$ of ERVC at resonant regime $\left(\mathrm{f}=\mathrm{f}_{\mathrm{res}}=\right.$ $50.3 \mathrm{~Hz}$ ) are shown in Fig. 6a. The amplitude of half-wave current pulse was $\mathrm{I}_{\mathrm{M}}=1.2 \mathrm{~A}$ while its time duration amounted $\tau=8 \mathrm{~m} \mathrm{sec}$. Under these conditions, the amplitude of output displacement of ERVC is made of $P_{m}=3 \mathrm{~mm}$, i.e., vibratory width $P_{p-p}=0.4 \mathrm{~mm}$. In Fig. $6 b$, there are given the same values but in case of amplitude of current half-wave pulses $\mathrm{I}_{\mathrm{M}}=0.45 \mathrm{~A}$ and their duration of $\tau=4 \mathrm{~m} \mathrm{sec}$. In this case, the amplitude of output displacement of ERVC is made of $P_{m}=0.2 \mathrm{~mm}$, i.e.,
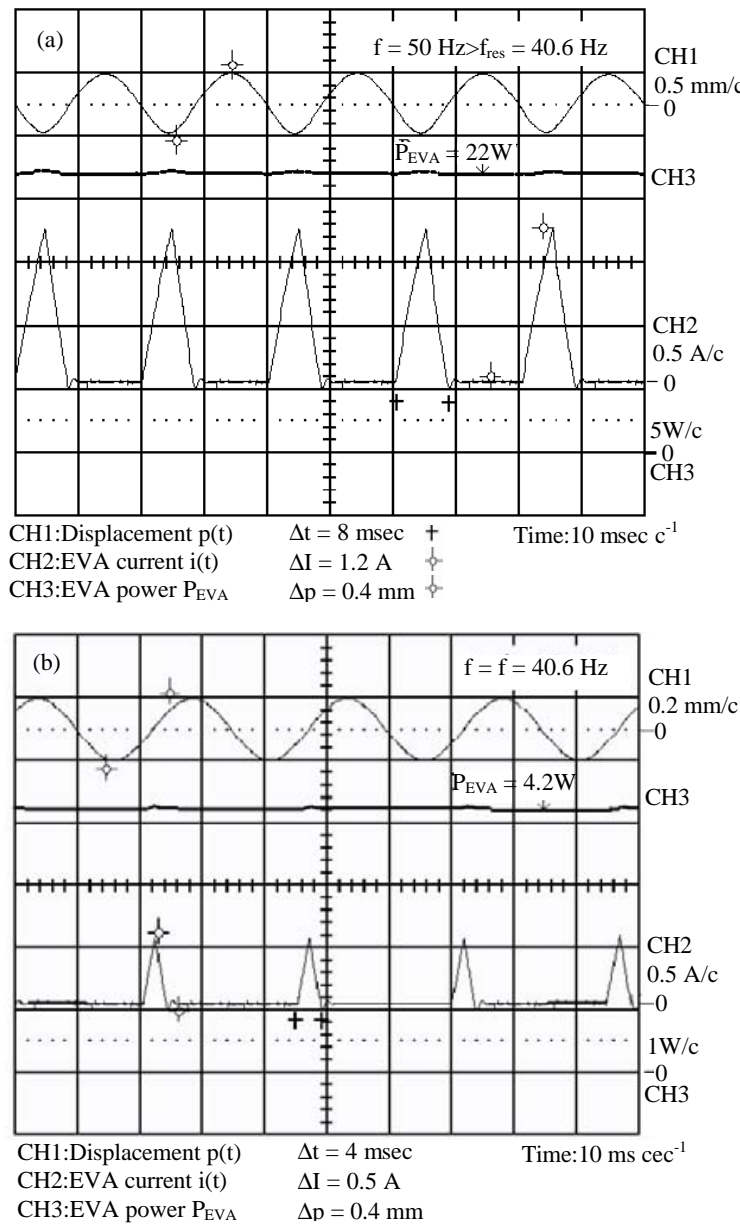

Fig. 7: Influence of tracking the mechanical resonant frequency on power consumption of EVA; a) super resonant mode $f>f_{\text {res }} ; b$ ) resonant mode $f=f_{\text {res }}$

vibratory width $P_{p-p}=0.4 \mathrm{~mm}$. The frequency control of ERVC is shown in Fig. 7. The oscilloscopic records show the influence of tracking mechanical resonant frequency of ERVC on power consumption of EVA coil. Oscilloscopic records of EVA current and output displacement of ERVC in case inconsistent excitation end mechanical resonant frequency are shown in Fig. 7a.

In this case, excitation frequency was higher than resonant frequency, i.e., $\mathrm{f}=50 \mathrm{~Hz}>\mathrm{f}_{\mathrm{res}}=40.6 \mathrm{~Hz}$. Vibratory width $\mathrm{P}_{\mathrm{p}-\mathrm{p}}=0.4 \mathrm{~mm}$ is obtained through triangle current pulses of amplitude $I_{M}=1.2 \mathrm{~A}$ and time duration of $\tau=8 \mathrm{~m} \mathrm{sec}$ where the power consumption of EVA was approximately $\mathrm{P}_{\mathrm{EVA}}=22 \mathrm{~W}$. Oscilloscopic records of EVA current and output displacement of ERVC in case of equality excitation and mechanical resonant frequency are shown in Fig. 7b. In this case, excitation frequency is equal of resonant frequency, i.e., $\mathrm{f}=40 \mathrm{~Hz} \approx \mathrm{f}_{\text {res }}=40.6 \mathrm{~Hz}$ $(\mathrm{m}=1.76 \mathrm{~kg})$. To obtain the vibratory width as in the 
previous case is required much less amplitude current, i.e., $\mathrm{I}_{\mathrm{M}}=0.5 \mathrm{~A}$ and less time duration, i.e., $\tau=4 \mathrm{~m} \mathrm{sec}$ where power consumptionof EVA is significantly reduced and under these conditions amounted to approximately $\mathrm{P}_{\mathrm{EVA}}=$ $4.2 \mathrm{~W}$. The results show that to achieve the same output value of the amplitude oscillation ERVC, required five times less power in the event resonant regime $\left(f=f_{r e s}\right)$ compared to the super resonant regime $\left(f>f_{\text {res }}\right)$.

\section{CONCLUSION}

The request for optimum operation of ERVC drive in resonant range is persistently in modern industrial and technological processes: conveying, processing and dosing of bulk materials. Electromagnetic Vibratory Actuator (EVA) is due to its reliability and low cost is increasingly being used as a source of excitation force in drives when require a small displacement and a relatively high accelerations of conveying material. The research presented in this study has shown that it is possible to achieve significant energy efficiency of ERVC drive if it is amplitude and frequency controlled by means a switching power converter. As a qualitative measure of energy efficiency was adopted power consumption of EVA coil. The experimental results presented in this srudy has shown that the resonant mode of vibration conveyor with electromagnetic excitation is very advantageous since then consumes the least energy to maintain the system in a state of oscillation. It was noted that the tracking algorithm mechanical resonant frequency can achieve power savings of up to five times. This has a great importance in process systems which employ a number of these drives.

\section{REFERENCES}

Despotovic, Z. and A. Ribic, 2009. Low frequency IGBT converter for control exciting force of electromagnetic vibratory conveyors. Int. Symp. Power, Electron., T11.8: $1-5$.

Despotovic, Z. and Z. Stojiljkovic, 2005. PSPICE simulation of two mass vibratory conveying system with electromagnetic drive. Proceedings of the International Conference Computer as a Tool, November 21-24, 2005, Belgrade, pp:1509-1512.
Despotovic, Z. and Z. Stojiljkovic, 2007. Power converter control circuits for two-mass vibratory conveying system with electromagnetic drive: Simulations and experimental results. IEEE, Trans. Ind. Electron., 54: 453-466.

Dewan, S.B. and A. Straughen, 1975. Power Semiconductor Circuits. John Wiley and Sons Ltd., New York, ISBN: 047121180X, 9780471211808, Pages: 523.

Doi, T., K. Yoshida, Y. Tamai, K. Kono, K. Naito and T. Ono, 1999. Modeling and feedback control for vibratory feeder of electromagnetic type. J. Rob. Mechatron., 11: 367-373.

Doi, T., K. Yoshida, Y. Tamai, K. Kono, K. Naito and T. Ono, 2001. Feedback control for electromagnetic vibration feeder Int. J, Ser. C,. 44: 44-52.

Dyr, T. and P. Wodzinski, 2002. Model particle velocity on a vibrating surface. Physicochem. Prob. Mineral. Process., 36: 147-157.

Goncharevich, L.F., K.V. Frolov and E.I. Rivin, 1990. Theory of Vibratory Technology. Hemisphere Publishing Corporation, New York, ISBN: 13:9780891167006, Pages: 350.

Mohan, N., T.M. Undeland and W.P. Robbins, 1995. Power Electronics-Converters, Applications and Design. John Wiley and Sons, New York.

Parameswaran, M.A. and S. Ganapahy, 1979. Vibratory conveying-analysis and design: a review. Mech. Mach. Theory, 14: 89-97.

Rashid, M.H., 1988. Power Electronics. Prentice-Hall Englewood Cliffs, New York.

Ribic, A.I. and Z. Despotovic, 2010. High-performance feedback control of electromagnetic vibratory feeder. IEEE, Trans. Ind. Electron., 57: 3087-3094.

Sloot, E.M. and N.P. Kruyt, 1996. Theoretical and experimental study of the conveyance of granular materials by inclined vibratory conveyors. Powder Technol., 87: 203-210.

Sokolov, I.J., V.I. Babitsky and N.A. Halliwell, 2007. Autoresonant vibro-impact system with electromagnetic excitation. J. Sound Vibr., 308: 375-391.

Soto-Yarritu, G.R. and A.A. Martinez, 2001. Computer simulation of granular material: Vibrating feeders. Powder Handling Process., 13: 181-185. 